\title{
Fine Structure of Shear Bands Formed during Hot Deformation of Two Austenitic Steels
}

\author{
Pavel Cizek, Fang Bai, W. Mark Rainforth and John H. Beynon \\ Institute for Microstructural and Mechanical Process Engineering: The University of Sheffield (IMMPETUS), \\ Department of Engineering Materials, The University of Sheffield, Mappin Street, Sheffield S1 3JD, United Kingdom
}

\begin{abstract}
Shear bands formed during both cold and hot plastic deformation have been linked with several proposed mechanisms for the formation of ultrafine grains. The aim of the present work was to undertake a detailed investigation of the microstructural and crystallographic characteristics of the shear bands formed during hot deformation of a $22 \mathrm{Cr}-19 \mathrm{Ni}-3 \mathrm{Mo}$ (mass $\%$ ) austenitic stainless steel and a Fe-30 mass $\% \mathrm{Ni}$ based austenitic model alloy. These alloys were subjected to deformation in torsion and plane strain compression (PSC), respectively, at temperatures of $900^{\circ} \mathrm{C}$ and $950^{\circ} \mathrm{C}$ and strain rates of $0.7 \mathrm{~s}^{-1}$ and $10 \mathrm{~s}^{-1}$, respectively. Transmission electron microscopy and electron backscatter diffraction in conjunction with scanning electron microscopy were employed in the investigation. It has been observed that shear bands already started to form at moderate strains in a matrix of pre-existing microbands and were composed of fine, slightly elongated subgrains (fragments). These bands propagated along a similar macroscopic path and the subgrains, present within their substructure, were rotated relative to the surrounding matrix about axes approximately parallel to the sample radial and transverse directions for deformation in torsion and PSC, respectively. The subgrain boundaries were largely observed to be non-crystallographic, suggesting that the subgrains generally formed via multiple slip processes. Shear bands appeared to form through a co-operative nucleation of originally isolated subgrains that gradually interconnected with the others to form long, thin bands that subsequently thickened via the formation of new subgrains. The observed small dimensions of the subgrains present within shear bands and their large misorientations clearly indicate that these subgrains can serve as potent nucleation sites for the formation of ultrafine grain structures during both subsequent recrystallisation, as observed during the present PSC experiments, and phase transformation.
\end{abstract}

(Received January 21, 2004; Accepted February 9, 2004)

Keywords: shear bands, hot deformation, ultrafine grains, dislocation substructure, recrystallisation, electron backscatter diffraction, transmission electron microscopy

\section{Introduction}

Shear bands are at the heart of several proposed mechanisms for the formation of ultrafine grains. This is primarily because they are at the right length scale for nucleation of such fine grains and also because they appear under the deformation conditions that seem to be necessary for ultrafine grains to be formed. The nucleation of ultrafine grains from shear bands developed by cold rolling of an austenitic stainless steel (type 310S) was proposed by Morikawa et al. ${ }^{1)}$ where the shear bands were observed in the regions of a dense twin-matrix lamellar structure. Nucleation of recrystallised grains along shear bands formed by cold rolling was observed in $\mathrm{Fe}-3 \% \mathrm{Si}$ single crystals ${ }^{2)}$ where two types of shear band were found, distinguished by their orientation to the macroscopic rolling direction. That shear bands orient themselves preferentially to the macroscopic deformation axes rather than crystallographically is one of their key characteristics. Maintaining a fixed angle to a deformation axis requires continuous rotation of the interior of a shear band. Such rotations have been studied by Wagner et al. ${ }^{3)}$ who catalogued a multiplicity of slip activity in cold rolled aluminium, $\mathrm{Cu}-11.8 \% \mathrm{Al}$ and copper single crystals.

The formation of shear bands and their potency for nucleation of recrystallisation is retained during elevated temperature deformation of steels. ${ }^{4,5)}$ Barnett $^{6)}$ has shown that during warm (i.e. ferritic) rolling of a titanium interstitial-free steel, in-grain shear banding provided sites for nucleation of recrystallisation with a prevalence of ND fibre grains (i.e. those with a $\langle 111\rangle$ axis parallel to the sheet normal). It was concluded that the relative absence of ingrain shear bands in a warm rolled low-carbon steel might explain the dearth of ND fibre grains in the recrystallisation texture of this steel. ${ }^{6)}$ Hurley et al. ${ }^{7)}$ suggested the straininduced transformation rolling (SITR) mechanism for producing ultrafine grains whereby intragranular deformation features produced by the shear become active sites for ferrite nucleation. SITR has been shown to work for a wide range of steels $^{8)}$ implying a robust ferrite nucleation mechanism. Since a large austenite grain size is required for this approach, classical grain boundary nucleation of ferrite plays only a minor role ${ }^{9}$ ) and intragranular nucleation must dominate. Hurley et al. ${ }^{10)}$ used electron backscatter diffraction to show that ferrite was nucleating on shear bands in austenite, which was demonstrated by the observed crystallographic texture relationship.

Since shear bands are so important for the nucleation of ultrafine grains, either by recrystallisation or phase transformation, encouraging their formation will assist the development of ultrafine structures. At present, there has been only very limited information available on the detailed characteristics of the fine structure of shear bands, in particular for the case of hot deformation. More such data are needed in order to obtain a better understanding of the shear band formation mechanism, which would help optimise the strain paths designed to produce ultrafine microstructures. The aim of the present work was to undertake a detailed investigation of the fine structure of shear bands formed during hot deformation of two austenitic steels, a high alloy stainless steel and a Fe30 mass $\% \mathrm{Ni}$ based alloy. Both these alloys retain a stable austenitic structure after cooling to room temperature, thus allowing the high-temperature deformation microstructures to be readily characterised. The latter alloy has been widely used to simulate the hot forming behaviour of low carbon steel austenite. ${ }^{11)}$ A novelty of the present investigation is partly in the relatively small plastic strains imposed, showing 
that shear bands appeared perhaps surprisingly early in the deformation of austenite. This also facilitated a study of the nucleation stages of shear band formation.

\section{Experimental Procedures}

Both a 22Cr-19Ni-3Mo (mass\%) austenitic stainless steel and a $\mathrm{Fe}-30$ mass $\% \mathrm{Ni}$ based model alloy were used in the investigation. The high alloy steel had the chemical composition of 0.02 mass \% C, $0.99 \% \mathrm{Mn}, 0.55 \% \mathrm{Si}, 0.023 \% \mathrm{P}$, $0.016 \% \mathrm{~S}, 21.54 \% \mathrm{Cr}, 18.61 \% \mathrm{Ni}, 2.93 \% \mathrm{Mo}$ and the balance Fe. This steel was subjected to deformation in hot torsion. The corresponding mean starting recrystallised grain size was about $60 \mu \mathrm{m}$. Solid specimens with a gauge length of $50 \mathrm{~mm}$ and a diameter of $6 \mathrm{~mm}$ were deformed at a strain rate of $0.7 \mathrm{~s}^{-1}$ at $900^{\circ} \mathrm{C}$ to equivalent von Mises strain levels of approximately $0.2,0.5,0.8$ and 1.1 and rapidly quenched in water. Transmission electron microscopy (TEM) was employed to examine thin foils obtained from tangential sections of the torsion rod specimens, at a depth of $1 \mathrm{~mm}$ under the surface. A Philips CM 20 microscope operated at $200 \mathrm{kV}$ was used in the examination.

The model alloy had the chemical composition of 0.092 mass\% C, $0.19 \% \mathrm{Si}, 1.67 \% \mathrm{Mn}, 0.009 \% \mathrm{P}, 0.003 \%$ $\mathrm{S}, 30.3 \% \mathrm{Ni}, 1.51 \% \mathrm{Mo}$ and the balance Fe. This alloy was deformed in hot plane strain compression (PSC). Specimens with the dimensions of $50 \times 30 \times 10 \mathrm{~mm}$ were preheated at $1250^{\circ} \mathrm{C}$ for $15 \mathrm{~min}$ and deformed at a temperature of $950^{\circ} \mathrm{C}$ and a strain rate of $10 \mathrm{~s}^{-1}$ to true strain levels of approximately $0.2,0.4,0.6$ and 0.7 . The mean recrystallised grain size obtained after preheating was about $120 \mu \mathrm{m}$. After being removed from the servohydraulic test machine, PSC specimens were quenched in water. The time required for the specimens to cool down to room temperature was approximately $50 \mathrm{~s}$. Investigation of the grain structure, crystallographic texture and dislocation substructure was undertaken in the central areas of PSC specimens on sections containing the elongation (ED) and normal (ND) sample directions, using the quantitative light microscopy, electron backscatter diffraction (EBSD) and TEM techniques. EBSD study was performed using an FEI XL30 Sirion field emission gun scanning electron microscope, equipped with the HKL Technology EBSD attachment, operated at $15 \mathrm{kV}$. The corresponding data acquisition and processing were carried out using the HKL Channel 5 software. TEM examination of thin foils was performed using a Philips EM 420 microscope operated at $120 \mathrm{kV}$.

\section{Experimental Results}

\subsection{High alloy austenitic steel deformed in hot torsion}

Figure 1 shows the flow curve obtained for the hot torsion of the $22 \mathrm{Cr}-19 \mathrm{Ni}-3 \mathrm{Mo}$ steel. The curve was characterised by reaching the steady-state flow stress regime at a strain of about 0.4 , which is indicative of the dynamic recovery softening mechanism, and no recrystallised grains were found in the microstructure. Shear band nuclei were first detected within several grains at a strain of 0.5 using TEM. At this strain level, the microstructure was dominated by "microbands" 12) that occupied the interior of a majority of

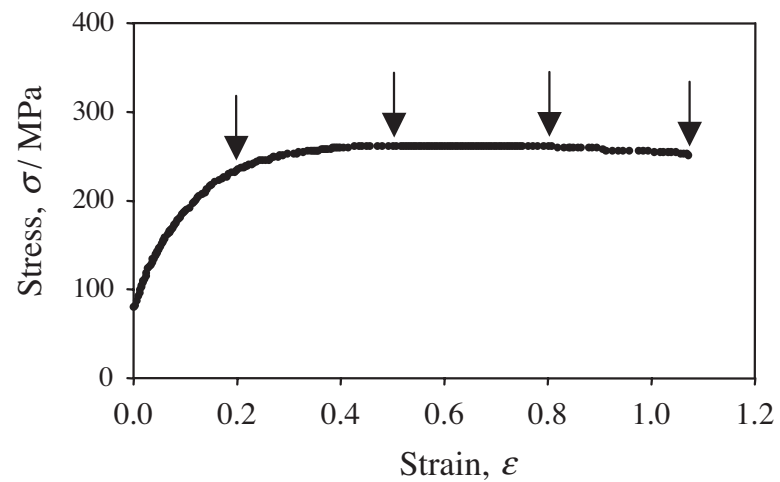

Fig. 1 Flow curve obtained for hot torsion of the $22 \mathrm{Cr}-19 \mathrm{Ni}-3 \mathrm{Mo}$ steel (arrows indicate strain levels at which a detailed TEM investigation was undertaken).

grains. The planar dislocation walls delineating microbands were frequently aligned approximately parallel to one of the $\{111\}$ crystallographic slip planes and the corresponding misorientation angles were found to be largely less than two degrees. Shear bands nucleated on the background of preexisting microbands as originally isolated well-recovered subgrains (or small groups of subgrains), being about $0.5 \mu \mathrm{m}$ in diameter, macroscopically aligned along the path in which these bands propagated across the grain [Fig. 2(a)]. The subboundaries parallel to the shear band propagation paths were largely not aligned with any of the $\{111\}$ slip planes and thus generally appeared non-crystallographic. The relative misorientation profiles corresponding to the linescans performed in the width direction, perpendicular to the shear band propagation paths, showed that misorientation angles across the sub-boundaries locally reached values as large as about $10^{\circ}$ already at the band nucleation stages [Fig. 2(b)]. The shear band nucleation was generally accompanied by noticeable shear displacements of the microband walls [see Fig. 2(a)]. The cumulative misorientation profiles for the above linescans showed that, when crossing the shear band, the accumulated misorientation angles displayed an increase to a peak value, followed by their subsequent gradual decrease towards the matrix orientation [Fig. 2(c)]. The formation of new subgrains thus appeared to be assisted by the stress fields generated by lattice rotations of the previously formed subgrains. From Fig. 2(c) it is also evident that the cumulative misorientation gradients introduced by nucleating shear bands were quite large, in this particular case locally reaching about $15^{\circ}$. These misorientation gradients were found to differ significantly in value along the shear band propagation path. This further demonstrates that the subgrains comprising the shear band were formed at different time intervals and thus underwent different amounts of crystal lattice rotation before merging together into a larger band. The rotation axis vectors, about which the subgrains rotated with respect to the surrounding matrix of microbands as well as relative to each other, were clustered around a common macroscopic direction, approximately parallel to the radial direction of the torsion rod [Fig. 2(d)]. When expressed in the crystal lattice coordinates, these vectors were scattered around a large area of the standard stereographic triangle, which indicates that they did not have a 

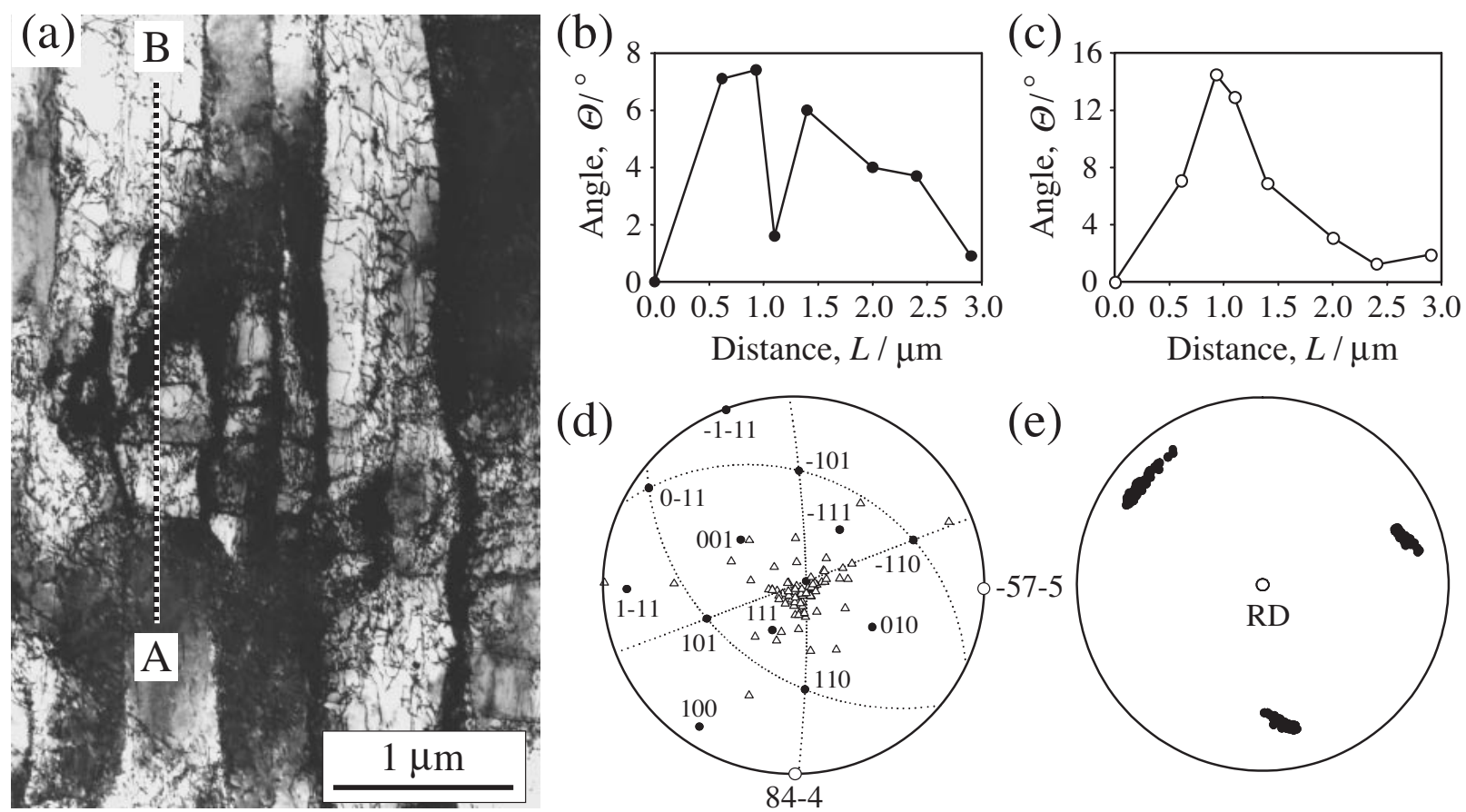

(d)

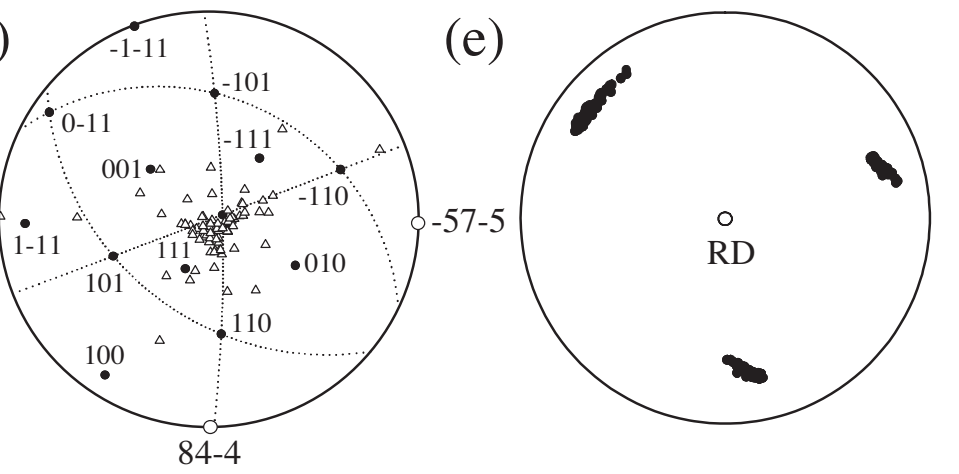

Fig. 2 Characteristics of the nucleating shear bands observed during hot torsion of the 22Cr-19Ni-3Mo steel at a strain of 0.5: (a) TEM bright-field micrograph of a shear band nucleating within the matrix of microbands and propagating in a horizontal direction; (b), (c) relative and cumulative misorientation angle distributions, respectively, along the line A-B indicated in (a); (d), (e) misorientation axis vectors expressed in a full inverse pole figure and orientations plotted in a $\{100\}$ direct pole figure, respectively, corresponding to the area shown in (a) (centres of the above pole figures approximately coincide with the radial direction (RD) of the torsion rod; the remaining two sample axes in (e) are arbitrary).

pronounced crystallographic preference. The observed subgrain rotations, largely occurring about the torsion rod radial direction, also manifested themselves by a characteristic azimuthal spread of the corresponding orientations plotted in a pole figure [Fig. 2(e)].

At strains of 0.8 and 1.1 , the shear bands were locally rather well developed on the microscopic scale, reaching maximum diameters of about $5 \mu \mathrm{m}$, and they remained confined within the grain interiors without being able to cross the original grain boundaries. It was difficult to observe these bands clearly using optical microscopy since they occupied only tiny areas of the grains. As a result, it was not possible to extract clear information on the macroscopic geometrical form of the shear bands. In order to obtain such information, sectioning experiments utilising significantly larger shear bands would be required. As illustrated in Fig. 3, the general characteristics of the relatively well-developed shear bands remained similar to those found for the bands being at early stages of their formation. Well-developed bands were again observed to be composed of well-recovered and highlymisoriented fine subgrains with diameters around $0.5 \mu \mathrm{m}$ [Fig. 3(a)]. The misorientations across sub-boundaries generally increased with strain [Fig. 3(b)] and the introduced cumulative misorientation gradients became rather large as the locally accumulated misorientation angles often reached about $20^{\circ}$ [Fig. 3(c)]. In parallel, shear displacements of the microband walls, accompanying shear band formation, also became more pronounced. In analogy to the nucleating bands, the subgrains comprising relatively well-developed shear bands rotated with respect to the surrounding matrix of microbands, as well as relative to each other, about noncrystallographic axes aligned approximately parallel with the radial direction of the torsion rod specimen [Fig. 3(d)]. This was also manifested by the characteristic azimuthal spread of the corresponding orientations in a pole figure [Fig. 3(e)].

\subsection{Model alloy deformed in hot plane strain compres- sion}

Figure 4 shows the flow curve obtained for the hot PSC of the Fe-30 mass $\% \mathrm{Ni}$ based model alloy. Although the shape of the curve displaying a stress peak is often taken as an indication of the dynamic recrystallisation softening mechanism, some macroscopic shearing of the PSC specimens occurring at strains of 0.6 and 0.7 appeared to have been largely responsible for the observed decrease in flow stress. As revealed using light microscopy and EBSD, the evolution of grain structure with increasing strain was characterised by a gradual increase in volume fraction of new recrystallised grains containing significant amounts of twins (see further below). A detailed TEM investigation indicated that these grains largely appeared to form statically in the finite time before quenching.

The observed evolution of the crystallographic texture of deformed grains, determined using the EBSD technique, was largely characterised by crystallite rotations towards the $\beta$ fibre, ${ }^{13)}$ connecting the Brass $\{011\}\langle 211\rangle$ and Copper $\{112\}\langle 111\rangle$ texture components, accompanied by rotations towards the Goss $\{011\}\langle 100\rangle$ and $S\{123\}\langle 634\rangle$ components [Fig. 5(a)]. When reaching a strain of 0.6 , characterised by macroscopic shearing, the fraction of recrystallised grains 

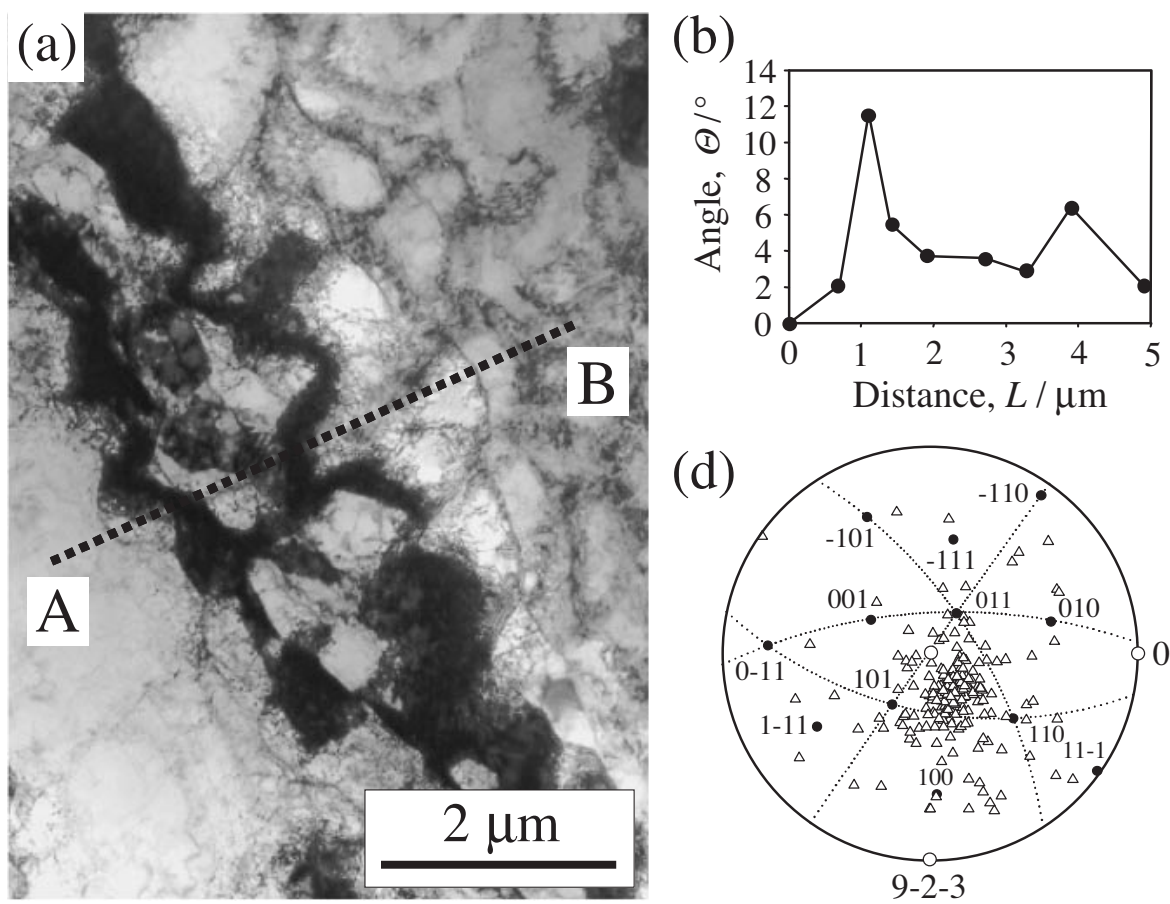

(c)

(d)

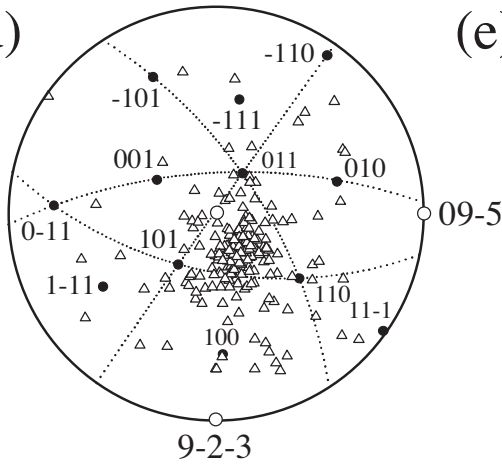

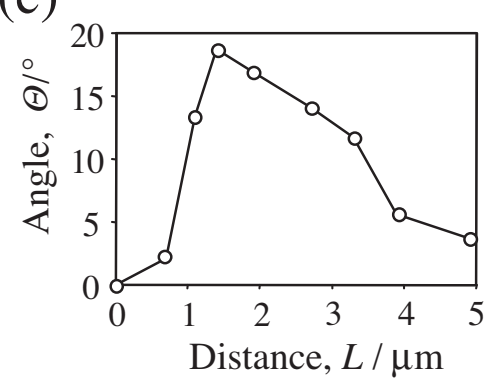

(e)

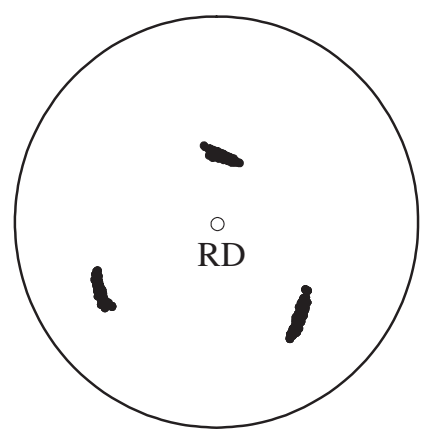

Fig. 3 Characteristics of the relatively well-developed shear bands observed during hot torsion of the $22 \mathrm{Cr}-19 \mathrm{Ni}-3 \mathrm{Mo}$ steel at a strain of 1.1: (a) TEM bright-field micrograph of a shear band composed of fine recovered subgrains; (b), (c) relative and cumulative misorientation angle distributions, respectively, along the line A-B indicated in (a); (d), (e) misorientation axis vectors expressed in a full inverse pole figure and orientations plotted in a $\{100\}$ direct pole figure, respectively, corresponding to an area close to that shown in (a) (centres of the above pole figures approximately coincide with the radial direction (RD) of the torsion rod; the remaining two sample axes in (e) are arbitrary).

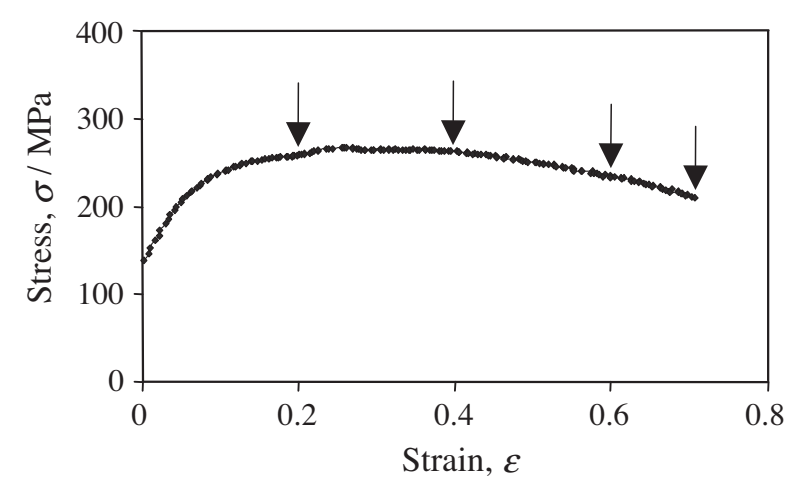

Fig. 4 Flow curve obtained for hot PSC of the Fe-30 mass $\% \mathrm{Ni}$ based model alloy (arrows indicate strain levels at which a detailed TEM and EBSD investigation was undertaken).

exceeded $90 \%$ and their crystallographic texture was extremely weak [Fig. 5(b)], similar to that obtained for the recrystallised grain at the lower strain levels. The corresponding recrystallised grain size distribution displayed a bimodal character, being composed of both very fine and moderately sized grains. The mean diameter of the fine recrystallised grains was about $4 \mu \mathrm{m}$ and they appeared to be arranged in band-like segments inclined to the extension direction at angles between 20 and $40^{\circ}$ (Fig. 6). This indicates that these grains originated from large shear bands developed during hot deformation to this strain level, as expected for the observed macroscopic shearing of the corresponding PSC specimen. The nucleation of the very fine grains appeared to be followed by profuse annealing twin formation and the grains might have also undergone some coarsening during the finite time interval before quenching. Following a strain of 0.7 the microstructure was completely recrystallised, maintaining similar characteristics to those observed at a strain level of 0.6. This reflects the acceleration of the static recrystallisation during cooling due to the larger imposed strain.

Shear band formation within the deformed grains was first locally observed at a strain of 0.4 using both the TEM and EBSD techniques. At this strain level, the microstructure was predominantly composed of well-recovered elongated subgrains, frequently arranged in extended microbands, ${ }^{12)}$ the boundaries of which were often aligned approximately parallel to one of the $\{111\}$ crystallographic slip planes. The corresponding misorientation angles across sub-boundaries were found to be largely in the range between $1^{\circ}$ and $5^{\circ}$. The observed deformation substructure characteristics thus appeared to be rather similar to those found for deformation of the high alloy steel in hot torsion. Nevertheless, subgrains formed in the model alloy generally seemed to be significantly more recovered. In analogy to the hot torsion, shear bands developed during PSC were observed to form on the background of pre-existing microbands as strings of wellrecovered subgrains, aligned along the common macroscopic path [Fig. 7(a)]. These bands were locally bent and the corresponding straight segments were inclined to the elongation direction at a wide range of angles. Nucleating bands, characterised by comparatively smaller misorientations, largely appeared to display inclination angles between $30^{\circ}$ 
(a)
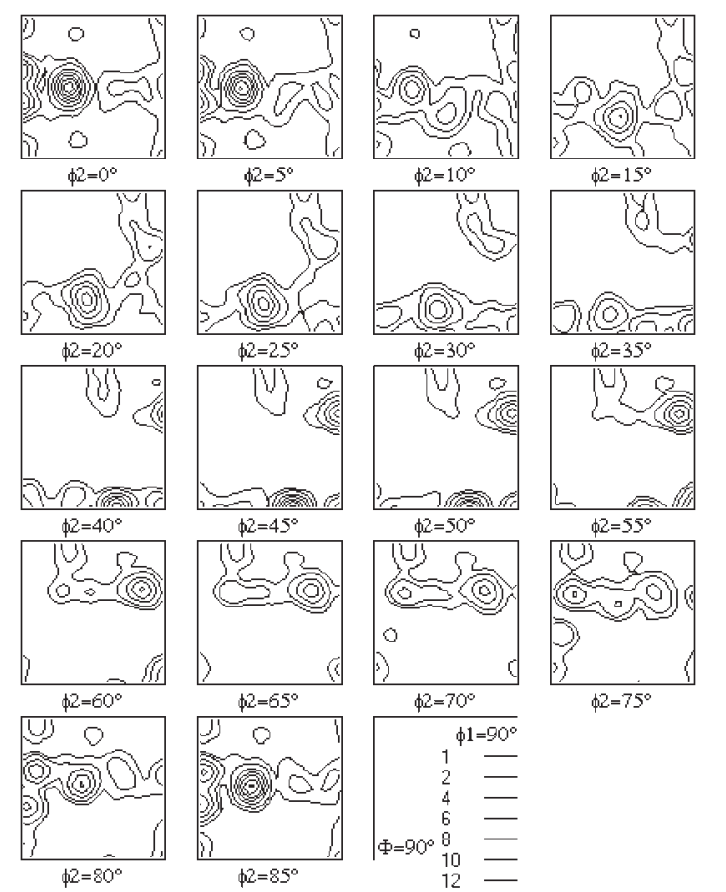

(b)
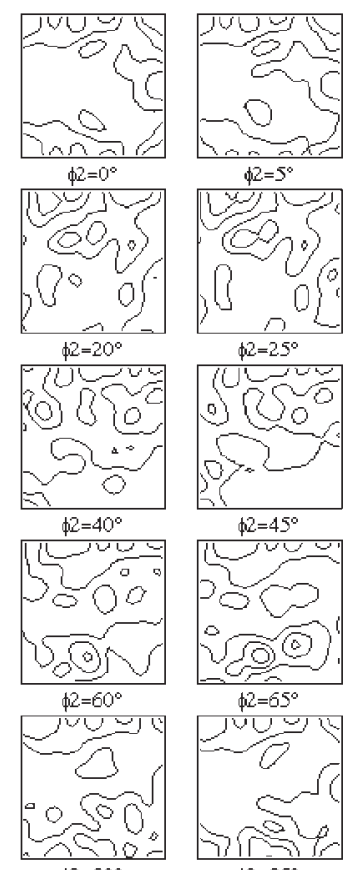

$+2=80^{\circ}$
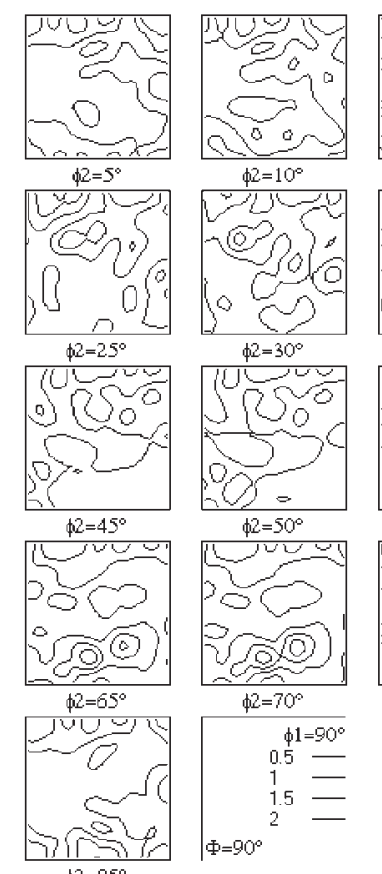

$+2=70$

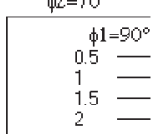

$\phi 2=85^{\circ}$

walls and did not seem to cross the grain boundaries. Several very fine, presumably recrystallised, grains completely enclosed by high-angle boundaries (misorientations above $15^{\circ}$ ) and having diameters less than $2 \mu \mathrm{m}$ appeared to be locally observed within these bands [see Fig. 7(a)]. The relative misorientation profiles, corresponding to the linescans performed across the shear bands in the width direction, showed that misorientation angles across the subboundaries locally reached values exceeding $15^{\circ}$, typical of high-angle boundaries, already for moderately developed bands [Fig. 7(b)]. The cumulative misorientation profiles for the above linescans were characterised by a progressive accumulation of misorientation angles to a peak value followed by their gradual decrease towards the matrix orientation [Fig. 7(c)]. From the above figure it is also evident that the cumulative misorientation gradients introduced by moderately developed shear bands were significant, in this particular case locally exceeding $40^{\circ}$. These misorientation gradients were found to differ significantly in value along the shear band propagation path. The rotation axis vectors, about which the subgrains rotated with respect to the surrounding matrix of microbands, were largely clustered around the sample transverse direction [Fig. 7(d)] and did not appear to display any pronounced crystallographic preference. The observed subgrain rotations, largely occurring about the sample transverse direction, also manifested themselves by a characteristic spread of the corresponding orientations plotted in a pole figure [Fig. 7(e)]. It is evident that such a texture spread was characterised in this particular case by rotations from the matrix orientation, close to the $\mathrm{S}$ ideal texture component, towards the Brass component dominating the central areas of shear bands. Nevertheless, no general information on the texture development accompanying the shear band formation could be obtained during the present study. It is clear that the shear band characteristics observed during hot PSC of the model alloy were remarkably similar to those obtained for hot torsion of the high alloy steel, described in the previous section.

\section{Discussion}

Shear bands observed in the present study propagated across grains along a similar macroscopic path. Moreover, the subgrains comprising these bands were found to rotate with respect to the surrounding matrix of microbands, as well as relative to each other, about non-crystallographic axes clustered around the sample radial and transverse directions for the hot torsion and PSC, respectively. This suggests that characteristics of the shear bands were largely governed by the macroscopically imposed deformation geometry, which is in excellent agreement with the published data. ${ }^{12,14,18)}$ Even at the largest applied strain of 1.1 in hot torsion, the volume fraction of shear bands was small and they appeared to remain restricted to the grain interiors without being able to traverse the original grain boundaries. Such bands are sometimes called "in-grain shear bands" or "micro-shear bands". It is difficult to predict whether the fine shear bands, observed in the present study, would grow to large dimensions and propagate across several grains if deforma-

tion continued to significantly larger strains. The sub-

and $40^{\circ}$ in correspondence with the published data. ${ }^{12,14,18)}$ In contrast, relatively well-developed bands displayed very low inclination angles and often became almost parallel with the longitudinal direction [see Fig. 7(a)]. This indicates that the latter bands, after being formed, might have undergone some rigid body rotation during straining. Shear bands generally caused noticeable shear offsets on the underlying microband 


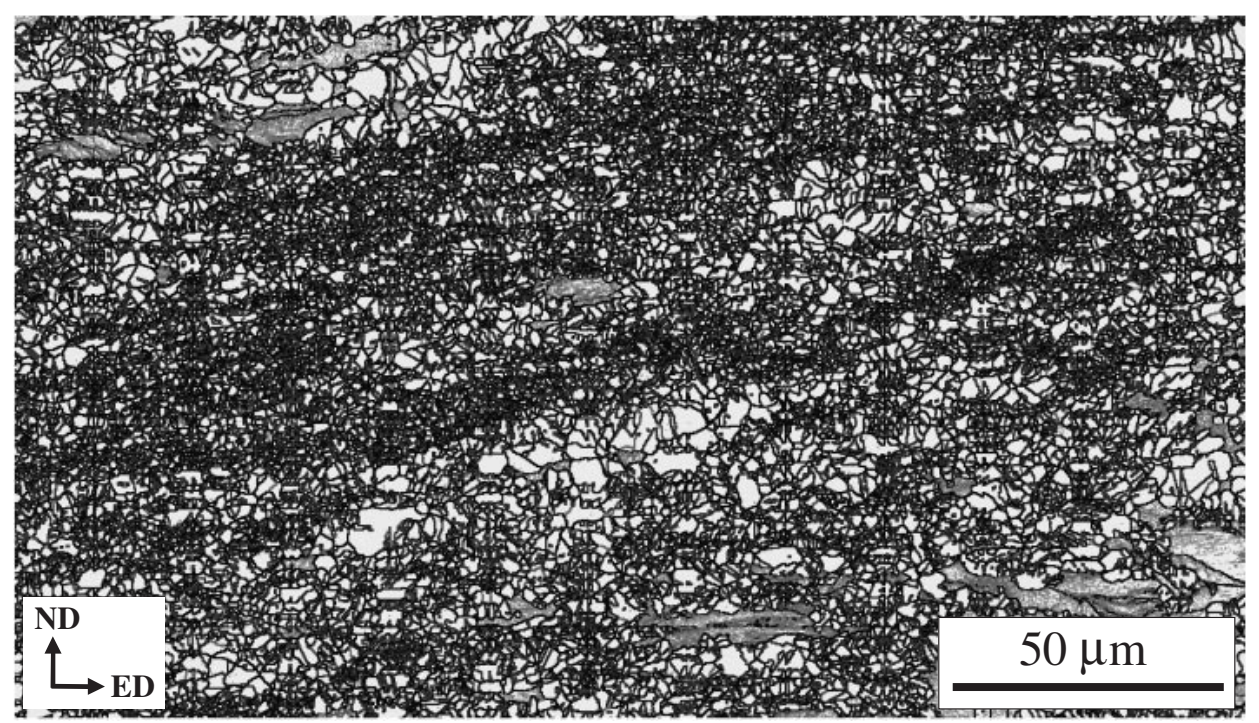

Fig. 6 EBSD orientation map corresponding to the Fe-30 mass $\%$ Ni based model alloy deformed in hot PSC to a strain level of 0.6 (elongated islands with substructure are the remnants of the original deformed grains; ED and ND denote the elongation and normal sample directions, respectively).

boundaries present within the shear bands were predominantly observed to be non-crystallographic, as they frequently did not seem to be aligned along any of the $\{111\}$ slip planes, and the subgrains thus largely appeared to form through the operation of multiple slip. This could be expected, bearing in mind that the subgrains, comprising shear bands formed in differently oriented grains within a polycrystalline sample, were all observed to be rotated relative to the surrounding matrix approximately about the common macroscopic direction. It has also been suggested in the literature ${ }^{14)}$ that multiple slip might frequently operate within shear bands and, in particular, might facilitate the transmission of these bands across grain boundaries.

The microstructural mechanism of shear band formation found in the present study does not seem to be consistent with the suggestions that these bands might form via coalescence of crystallographic coarse slip bands ${ }^{14)}$ or through nucleation on grain boundary steps and offsets. ${ }^{15)}$ Instead, the shear bands appeared to nucleate through the co-operative formation of discrete subgrains (slip domains) macroscopically aligned along the band propagation paths, as illustrated schematically in Fig. 8(a). These subgrains continuously increased their misorientations relative to the matrix of microbands during straining and gradually interconnected to form long, narrow bands that subsequently thickened via the formation of new subgrains [Fig. 8(b)]. The nucleation of new subgrains thus appeared to be assisted by the stress fields generated by lattice rotations of the previously formed subgrains. The subgrain formation was accompanied by noticeable shear displacements of the underlying microband walls, with the shear offsets being directly proportional to the subgrain rotation angle values. At later stages of the deformation process, relatively well-developed shear bands, characterised by ragged boundaries and composed of recovered subgrains having significant misorientations with respect to the surrounding matrix, were formed [Fig. 8(c)].

The observed macroscopic alignment of nucleation centres and their subsequent development into shear bands appear to be rather well explained by a concept of geometrical softening suggested by Harren et al. ${ }^{16)}$ The microstructural mechanism of shear band development, described above, appears to be similar to a concept suggested by Morii and Nakayama. ${ }^{17,18)}$ These authors proposed that a pre-existing lamellar microstructure, composed of either deformation twins or microbands, might be beneficial for shear band formation. In this microstructure, slip across the lamellar boundaries is inhibited, which enhances the tendency for the formation of localised slip inhomogeneities through necking and bending of the lamellae, creating local lattice curvatures. As observed in the present work and also by Morii and Nakayama, ${ }^{17)}$ these localised slip inhomogeneities appeared to have a form of small cells (subgrains), macroscopically aligned along the shear band propagation path (perhaps as a result of geometrical softening ${ }^{16)}$ ), which served as the band nucleation sites. It was systematically observed in the present study that, when crossing a shear band, the arrangement of the corresponding subgrain orientations displayed a cumulative misorientation pattern with a characteristic sigmoidal profile, in accord with the observed microstructural mechanism of shear band formation (see Fig. 8). A specific scatter of the shear band texture, observed after plane strain compression of $\mathrm{Cu}$ - and Al-based single crystals by Wagner et al., ${ }^{3)}$ Jasienski et al. ${ }^{19)}$ and Paul et al., ${ }^{20)}$ appears to be in good correspondence with the findings of the present study. A similar orientation scatter, manifesting itself as a pronounced asterism of the TEM diffraction spots obtained from shear band areas, was also reported for rolling of $\mathrm{Cu}$-based single crystals by Wróbel et al. ${ }^{21)}$

Several very fine, presumably recrystallised, grains fully enclosed by high-angle boundaries and having diameters less than $2 \mu \mathrm{m}$ were observed to form locally within shear bands during hot PSC of the model alloy at a strain of 0.4 [see Fig. 7(a)]. It is reasonable to suggest that these grains formed statically, in the finite time before quenching of the specimen, from higher-misoriented subgrains comprising the bands and were at early stages of their formation. The highly-misor- 

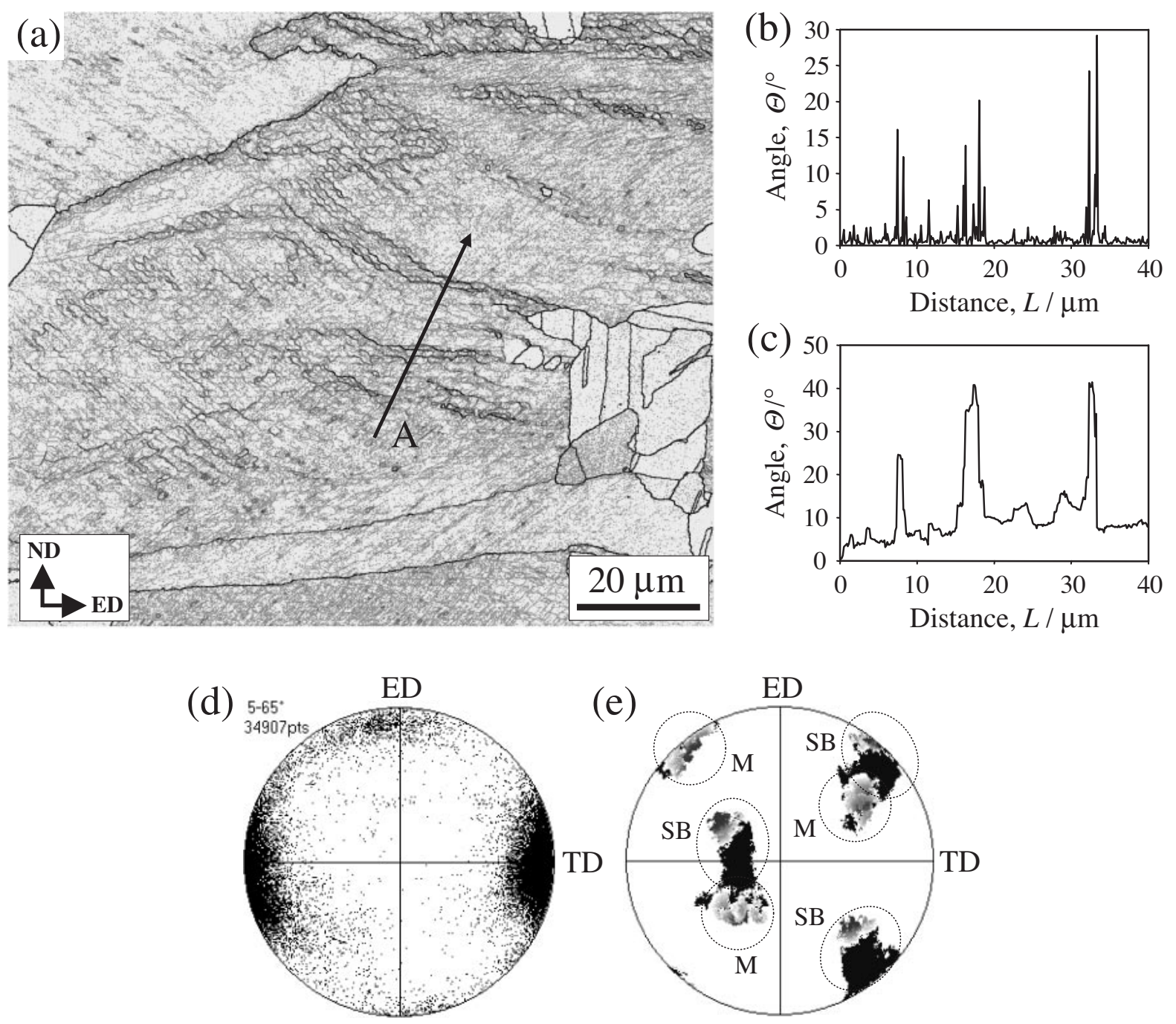

Fig. 7 EBSD analysis of the shear bands formed in the Fe-30 mass $\% \mathrm{Ni}$ based model alloy deformed in hot PSC to a strain level of 0.4: (a) orientation map showing the boundaries (ED and ND denote the elongation and normal sample directions, respectively); (b), (c) relative and cumulative misorientation angle distributions, respectively, along the line A crossing several shear bands in (a); (d), (e) misorientation axis vectors (for angles above $5^{\circ}$ ) expressed in the sample reference frame and orientations plotted in a $\{100\}$ direct pole figure, respectively, corresponding to the area shown in (a) (ED and TD denote the elongation and transverse sample directions, respectively; SB and M in (e) indicate the shear band and matrix orientations, respectively).

shear band propagation direction

(a)

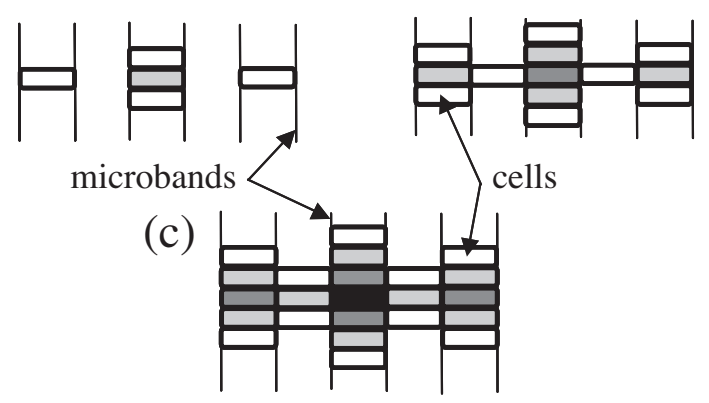

Fig. 8 Schematic representation of the observed microstructural mechanism of shear band formation: (a) nucleation stage; (b) growth stage; (c) well-developed shear band (grey levels are proportional to the rotation angles of individual subgrains (cells) relative to the matrix of microbands and corresponding shear displacements are perpendicular to the plane of the figure). iented fine subgrains present within the shear bands thus appear to have served as potential nuclei for the formation of ultrafine grains during subsequent recrystallisation. In an analogous manner, these subgrains could be expected to provide nucleation sites for ultrafine ferrite grains formed during subsequent phase transformation that were observed e.g. by Hurley et al.. ${ }^{7,10)}$ Mabuchi et al. ${ }^{8)}$ or Priestner and Ibrahim. ${ }^{9)}$ Recrystallised grains, which appeared to form along macroscopic shear bands, observed during hot PSC of the model alloy at a strain of 0.6 (see Fig. 6), displayed comparatively larger diameters than those found within the microscopic bands developed at a strain of 0.4. In this case it could be expected that the recrystallisation process would reach its completion very rapidly, due to a high stored energy in the large shear bands, and the recrystallised grains grown until their impingement might experience some further coarsening during the finite time before the quenching of the specimen. The nucleation of the above grains appeared to be rapidly followed by the profuse formation of annealing 
twins. The observed development of complex multiple twinning chains might account for the presence of random crystallographic texture found at strains of 0.6 [see Fig. 5(b)] and 0.7 . On the basis of the observed dimensions of the subgrains, comprising the shear bands, the limiting ultrafine grain size controlled by the subgrain size would appear to be in the range between $0.5-1 \mu \mathrm{m}$. It is interesting to speculate if this might be the limiting grain size that can be achieved by a shear band mechanism.

\section{Conclusions}

A detailed investigation of the fine structure of shear bands formed in both a $22 \mathrm{Cr}-19 \mathrm{Ni}-3 \mathrm{Mo}$ (mass \%) austenitic stainless steel and a Fe-30 mass \% Ni based austenitic model alloy, subjected to hot deformation in torsion and plane strain compression respectively, has been undertaken in the present work. It has been observed that the shear bands formed at moderate strains within a matrix composed of microbands and that their characteristics were predominantly governed by the macroscopically imposed deformation geometry. These bands appeared to be created through the co-operative formation of fine, highly-misoriented subgrains, largely resulting from the operation of multiple slip, serving as building blocks for the gradual development of shear bands with larger dimensions. These subgrains appeared to act as potential nuclei for the formation of ultrafine grains during subsequent recrystallisation or phase transformation.

\section{Acknowledgements}

The authors gratefully acknowledge the Engineering and Physical Sciences Research Council, UK for their financial support.

\section{REFERENCES}

1) T. Morikawa, T. Sato, K. Higashida and R. Onodera: Proc. 1st Int. Symp. on Ultrafine Grained Structures, ed. by S. Takaki and T. Maki, (Iron and Steel Inst. of Japan, 2001) pp. 160-163.

2) K. Ushioda and W. B. Hutchinson: ISIJ Int. 29 (1989) 862-867.

3) P. Wagner, O. Engler and K. Lücke: Acta Metall. Mater. 43 (1995) 3799-3812.

4) P. Cizek: Mater. Sci. Eng. A324 (2002) 214-218.

5) P. Cizek: Proc. Int. Conf. on Thermomechanical Processing: Mechanics, Microstructure and Control, ed. by E. J. Palmiere, M. Mahfouf and C. Pinna, (The University of Sheffield, 2003) pp. 289-295.

6) M. R. Barnett: ISIJ Int. 38 (1998) 78-85.

7) P. J. Hurley, P. D. Hodgson and B. C. Muddle: Scr. Mater. 40 (1999) 433-438.

8) H. Mabuchi, T. Hasegawa and T. Ishikawa: ISIJ Int. 39 (1999) 477485.

9) R. Priestner and A. K. Ibrahim: Mater. Sci. Technol. 16 (2000) 12671272.

10) P. J. Hurley, B. C. Muddle and P. D. Hodgson: Mater. Sci. Technol. 16 (2000) 1376-1379.

11) W. Charnock and J. Nutting: Met. Sci. Journ. 1 (1967) 123-127.

12) J. Hirsch, K. Lücke and M. Hatherly: Acta Metall. 36 (1988) 29052927.

13) V. Randle and O. Engler: Introduction to Texture Analysis - Macrotexture, Microtexture and Orientation Mapping, (Taylor \& Francis Ltd, London, 2000) pp. 111-112.

14) J. D. Embury, A. Korbel, V. S. Raghunathan and J. Rys: Acta Metall. 32 (1984) 1883-1894.

15) H. Inagaki: Z. Metallkde. 81 (1990) 474-483.

16) S. V. Harren, H. E. Dève and R. J. Asaro: Acta Metall. 36 (1988) 2435 2480 .

17) K. Morii and Y. Nakayama: Trans. JIM 22 (1981) 857-864.

18) K. Morii and Y. Nakayama: Trans. JIM 25 (1984) 276-283.

19) Z. Jasienski, T. Baudin, A. Piatkowski and R. Penelle: Scr. Mater. 35 (1996) 397-403.

20) H. Paul, J. H. Driver and Z. Jasienski: Acta Mater. 50 (2002) 815-830.

21) M. Wróbel, S. Dymek, M. Blicharski and S. Gorczyca: Z. Metallkde. 85 (1994) 415-425. 